\title{
A balancing act
}

\author{
Basic checkpoints and redundant modulatory mechanisms allow immune responses that are both efficient against \\ pathogens and safe to the host.
}

T he traditional view of an immune system activated by foreign molecular triggers or defined components of modified self is now evolving into that of a system tuned to detect additional alterations in tissue or organism homeostasis, including more elusive signals such as changes in metabolic rate, enzymatic activity or the location of various molecular components. Although the idea that the immune system has evolved to maintain the homeostasis of the organism is not new, the great variability of triggers, the versatility of the sensors and the complex interplay between effector and regulatory networks suggest that the ultimate function of an immune response, beyond the simple eradication of pathogens, is to allow a return to homeostasis. Additional complexities of the immune response, such as the interplay between basic and specialized cellular functions, suggest a highly balanced act that requires various checks as well as overlapping or redundant degrees of modulation that allow efficient immune responses with minimal collateral damage to the host.

In several specially commissioned articles, this Focus on Checks and Balances in the Immune System (http://www.nature.com/ni/ focus/checksandbalances) explores the cellular and molecular regulatory modules built in at every level of the system to fine-tune the immune response and limit the amount of tissue damage that can ensue and reviews recent progress in understanding how basic cellular processes such as cell metabolism are integrated into the more specialized function of immune cells to sustain efficient responses.

In a Focus Review, Ruoning Wang and Douglas Green discuss how cell metabolism interconnects with signaling events in cells of the immune system to provide the energy and functional requirements of immunological processes, such as rapid proliferation, differentiation and functional specialization. This is explored mainly in the context of metabolic reprogramming of $\mathrm{T}$ lymphocytes during activation. The authors discuss aspects of metabolic reprogramming and its consequences in terms of 'metabolic checkpoints', which they define as molecular mechanisms that sense metabolic status and, in turn, regulate cellular functions. Understanding these checkpoints has become key to the study of metabolic disorders, cancer and immune responses.

Because of the potent effector mechanisms deployed by an ongoing immune response, the potential to induce self-damage in the host is very high. Four commissioned pieces discuss various aspects of the balance between an effective immune response and avoiding excessive tissue damage. Ronald Germain authors a Commentary that places the regulatory modules embedded in an immune response in the larger context of systems organization and tackles the design principles of such mechanisms. This provocative piece puts forward concepts such as the fractal nature of the regulatory strategies that operate in a cell, a tissue or an organism and touches on considerations that are not so frequently discussed, such as the time-and-space subtleties in which particular factors function during an immune response.

Two Reviews discuss the regulatory mechanisms that restrain signaling though Toll-like receptors, as the main initiators of inflammation, and outline the soluble mediators that modulate and restrain inflammation. Peter Murray and Steve Smale define several interconnected 'strata' that restrain inflammation, from anatomy to the processing and secretion of proteins, and illustrate how anti-inflammatory factors such as IL-10 function in the context of these regulatory layers. Jacques Banchereau, Virginia Pasqual and Anne O'Garra present an updated discussion of how cytokines modulate inflammation in a Review that revisits old players, such as IL-2, IL-10 and TGF- $\beta$ and introduces the newcomers IL-27, IL-35 and IL-37.

In the final Review of the Focus, Romina Goldszmid and Giorgio Trinchieri present the pathogenic effects of immune responses or, as the authors introduce it, the 'price of immunity'. Their discussion focuses on the complex interactions between the immune response and the microbiota and the accumulating evidence that commensals have an active role in mucosal and skin pathologies associated with immune responses, but it also touches on the issue of collateral tissue and systemic damage caused by immunity to viruses and the ensuing susceptibility to superinfections, acute immunopathology, chronic inflammation and carcinogenesis that can be the consequence of such responses.

This Focus attempts to provide an integrated view of the immune system as a set of mechanisms designed to preserve the integrity of the organism against various homeostatic disturbances. Although pathogens represent the main source of homeostatic stress, and immune responses engage complex cellular and molecular mediators to fight viruses and microbes, the elimination or eradication of such pathogens may not always be compatible with the ultimate goal, which is the preservation of self. From this point of view, the maintenance of a suboptimal state, such as chronic infection, that still allows survival may be preferable to the ultimate pathogen eradication associated with extensive damage to self. Components of the organism have evolved various protective mechanisms against immune responses, and in most cases the immune response itself seems to follow the path of least harm. When these regulatory modules are lost or damaged, however, considerable pathology ensues. Understanding the principles and mechanisms of these regulatory modules is essential for the prevention and treatment of disease. 